Investigación original

\title{
Aproximación del rol de farmacéutico en la calidad de vida relacionada con la salud
} Approach to the role of the pharmacist in health related quality of life Edith TAFUR VALDERRAMA,Emilio GARCIA JIMENEZ..

\section{RESUMEN}

Mejorar la Calidad de Vida Relacionada con la Salud (CVRS) es un objetivo final de la Atención Farmacéutica.

El objetivo del estudio fue determinar el conocimiento, la actitud y la práctica de los farmacéuticos comunitarios sobre la evaluación y el uso de instrumentos para valorar la CVRS en sus pacientes.

El estudio fue descriptivo, observacional y basado en encuesta vía correo electrónico, durante marzo, abril y mayo del año 2005. Se envió un cuestionario sobre la calidad de vida y la practica farmacéutica a los farmacéuticos registrados en la base de datos del Centro de Atención Farmacéutica (CAF-

STADA), en total 1601. Se envió documentación científica sobre la CVRS y la práctica farmacéutica, el MOS SF 30; y un vínculo para responder un segundo cuestionario validado para recoger sus impresiones.

Se obtuvo que si bien los farmacéuticos comunitarios conocen poco sobre la calidad de vida relacionada con la salud; muestran una actitud positiva sobre el posible uso de instrumentos para evaluarla en el seguimiento farmacoterapéutico, reconociendo que necesitan entrenamiento y capacitación adicional; además evalúan subjetivamente la CVRS en sus pacientes, aunque no documentan la información en su mayoría. Estos resultados demuestran la existencia de un posible rol del farmacéutico comunitario en la evaluación de la CVRS, especialmente en enfermedades crónicas que afecten significativamente la CVRS; además esta evaluación puede ser incluida en el seguimiento farmacoterapéutico para valorar resultados en la mejora de calidad de vida como se define en el concepto de Atención Farmacéutica.

Palabras clave: Calidad de vida relacionada con la salud. Seguimiento Farmacoterapéutico. Atención Farmacéutica.
"Edith TAFUR VALDERRAMA. Químico Farmacéutico. Máster en Atención Farmacéutica. Becaria Programa Alßan.

Emilio GARCIA JIMÉNEZ. Doctor en Farmacia. Responsable del Centro de Atención Farmacéutica: CAFSTADA. Miembro Grupo de Investigación en Atención Farmacéutica. Universidad de Granada. (España)

\begin{abstract}
To improve health related quality of life (HRQoL) is a main objective of pharmaceutical care. The aim of the present study was to identify the knowledge, attitude and performance of community pharmacists on the assessment and the use of HRQoL tools.

The study was an observational based on an emailed survey during March to May 2005. A questionnaire focused on quality of life and pharmacy practice was sent to the pharmacists included in Pharmaceutical Care Center (CAFSTADA), a total of 1601. Literature on HRQoL and pharmacy practice and specifically MOS SF-36was sent to the pharmacists surveyed: then, a link to a webpage containing a validated questionnaire to gather their opinions.

Results showed that, although pharmacists are not much aware HRQoL, they are show a very positive attitude to use tools for evaluate patients' HRQoL. They recognize their need for supplementary education and training. Additionally, pharmacists state to be subjectively assessing HRQoL in their patients, without documenting it.

These results demonstrate the potential role of the pharmacist in assessing HRQoL. This assessment could be included in pharmacotherapy follow-up, in order to assess the outcomes on quality of life, as stated in original definition of pharmaceutical care.
\end{abstract}

Keywords: Health related quality of life. Pharmacotherapy follow-up. Pharmaceutical care.

\section{(Español)}

\section{INTRODUCCIÓN}

La calidad de vida relacionada con la salud (CVRS) evalúa el impacto de la enfermedad y el tratamiento en la vida del paciente, ${ }^{1-3}$ y va más allá de la recopilación y gradación de la presencia y severidad de los síntomas de la enfermedad evaluada o de los efectos adversos causados por el tratamiento administrado; e investiga como el paciente percibe y experimenta estas manifestaciones a diario, y como le afectan en el desarrollo de su vida habitual. ${ }^{4}$ Es una variable fundamental asociada al gasto sanitario, ${ }^{5}$ por lo que la información estandarizada sobre la CVRS 
mejoraría la calidad de la asistencia sanitaria. ${ }^{6} \mathrm{La}$ CVRS se evalúa mediante cuestionarios con referencia al inicio de la enfermedad, ${ }^{7}$ su diagnóstico y a los cambios de síntomas a través del tiempo. ${ }^{8-10}$ Debido a que el Seguimiento Farmacoterapéutico es una estrategia para mejorar y lograr el mayor beneficio de la farmacoterapia y persigue como resultado mejorar la CVRS del paciente,$^{11-13}$ es necesario evaluar la influencia de este en la CVRS; es necesario que los farmacéuticos sean capaces de evaluar críticamente la CVRS para la formulación de decisiones clínicas; además la evidencia de que los farmacéuticos pueden influir positivamente en los resultados de la salud contribuirá a expandir el rol de la profesión en el cuidado de la salud. Existe muy poca experiencia en la evaluación formal de la CVRS por los farmacéuticos; existiendo barreras conceptuales, metodológicas, de actitud y de práctica, para que los farmacéuticos adopten este rol; los farmacéuticos muestran mucho interés en utilizar instrumentos para evaluar CVRS (Bentley y $\mathrm{col})^{14}$. En las intervenciones farmacéuticas el cuestionario Medical Outcomes Study Short Form (SF-36) y sus variaciones ha sido el único instrumento utilizado, ${ }^{15}$ La calidad de vida en asmáticos mejoró después del seguimiento farmacoterapéutico. ${ }^{16}$ La CVRS mejoró a los tres meses de abstinencia ${ }^{17}$ en un programa de intervención farmacéutica para la cesación de fumar.

El objetivo de este estudio es determinar el conocimiento, la actitud y la práctica de los farmacéuticos comunitarios sobre la evaluación y el uso de instrumentos para valorar la CVRS de sus pacientes.

\section{MÉTODOS}

El estudio fue observacional, descriptivo y transversal, realizado durante marzo, abril y mayo del año 2005. La muestra la formaron los farmacéuticos comunitarios inscritos $(\mathrm{N}=1601)$ en la base de datos del Centro de Atención Farmacéutica (CAF-STADA), a quienes se les envió, vía correo electrónico, un cuestionario validado y dirigido a obtener datos sobre la actitud, el conocimiento y la evaluación de la Calidad de Vida Relacionada con la Salud, y las opiniones sobre los instrumentos para evaluar la CVRS. Se envió documentación científica sobre la CVRS y la práctica farmacéutica, y un instrumento para evaluar la CVRS, traducido y validado al español el MOS SF-36 (Medical Outcomes Study Short Form 36 ítem). En un segundo cuestionario, también validado, se recogieron las impresiones de los encuestados sobre la documentación y el instrumento entregado.

Los datos se procesaron con el programa SPSS v12 para Windows $X p$, se realizó una distribución de frecuencias para las variables cualitativas, para las variables cuantitativas se utilizaron las medidas de tendencia central (Me, Mo) y medidas de dispersión (Varianza y $\mathrm{DE})$, además de resúmenes numéricos.

\section{RESULTADOS}

Las características demográficas se muestran en la tabla 1 , donde más de la mitad $(67,1 \%)$ de los encuestados había leído o escuchado sobre la calidad de vida relacionada con la salud, antes de leer la definición dada en el cuestionario (tabla 2). EL $74 \%$ indica que el concepto de CVRS estuvo presente en los estudios universitarios. La autoevaluación sobre el conocimiento de la CVRS en una escala tipo likert del 1 al 5 , fue de muy poco a algo el conocimiento en su mayoría (tabla 3 ). La mayoría $(38,5 \%)$ considera que se necesita mucho conocimiento para evaluar la CVRS en sus pacientes.

\begin{tabular}{|l|l|}
\hline \multicolumn{2}{|l|}{ Tabla 1. Características demográficas } \\
\hline Característica & $\%$ \\
\hline Edad (años) Me 40,31 (8,3) & \\
\hline$\leq 32$ & 20,3 \\
\hline $33-40$ & 25,7 \\
\hline$\geq 41$ & 54 \\
\hline Género & \\
\hline Mujeres & 35,1 \\
\hline Hombres & 64,9 \\
\hline Farmacéutico & \\
\hline Titular & 65,8 \\
\hline Copropietario & 5,3 \\
\hline Adjunto & 21,1 \\
\hline Otros & 7,8 \\
\hline Otros: sustituto, en practicas \\
\hline
\end{tabular}

Tabla 2. Donde escuché el concepto de Calidad de Vida Relacionada con la Salud

\begin{tabular}{|c|c|}
\hline Donde & $\%$ \\
\hline $\begin{array}{l}\text { Publicaciones } \\
\text { farmacia }\end{array}$ & 33,33 \\
\hline Cursos de formación continua & 29,37 \\
\hline Congresos de farmacia & 17,46 \\
\hline Farmacia clínica & 9,52 \\
\hline Otros & 10,31 \\
\hline Total & 100,00 \\
\hline \multicolumn{2}{|c|}{$\begin{array}{l}\text { Otros: Revistas de Medicina, Literatura de Industria } \\
\text { Farmacéutica, clases universitarias, cursos a distancia } \\
\text { de CAF-STADA páginas web de farmacia, cursos de } \\
\text { LIFARA }\end{array}$} \\
\hline
\end{tabular}

\begin{tabular}{|l|c|c|}
\hline $\begin{array}{l}\text { Tabla 3. Conocimiento sobre la Calidad de Vida } \\
\text { Relacionada con la Salud }\end{array}$ \\
\hline Escala & $\begin{array}{c}\text { Conocimiento } \\
\text { actual de } \\
\text { CVRS (\%) }\end{array}$ & $\begin{array}{c}\text { Conocimiento } \\
\text { para evaluar } \\
\text { CVRS (\%) }\end{array}$ \\
\hline Ningún conocimiento & 19,7 & 0 \\
\hline $\begin{array}{l}\text { Muy poco } \\
\text { conocimiento }\end{array}$ & 36,8 & 34,6 \\
\hline Conoce algo & 38,3 & 0 \\
\hline Conoce & 2,6 & 26,9 \\
\hline Conoce mucho & 1,3 & 38,5 \\
\hline Perdidos & 1,3 & 0 \\
\hline Total & 100 & 100 \\
\hline
\end{tabular}

Antes de leer el MOS SF-36, el 81,6\% contestó que un instrumento sistemático para recoger información sobre la CVRS sería útil en la práctica profesional, y menos del 3\% respondieron que no sería útil. Así mismo la mayoría $(80,3 \%)$ respondió que si utilizaría un instrumento para evaluar la CVRS en sus pacientes. Después de leer la documentación científica sobre CVRS y la práctica farmacéutica, el MOS SF-36; la mayoría $(92,4 \%)$ 
respondió que este instrumento sería útil en su práctica profesional, disminuyendo el porcentaje de farmacéuticos dispuestos a utilizarlos (figura 1).
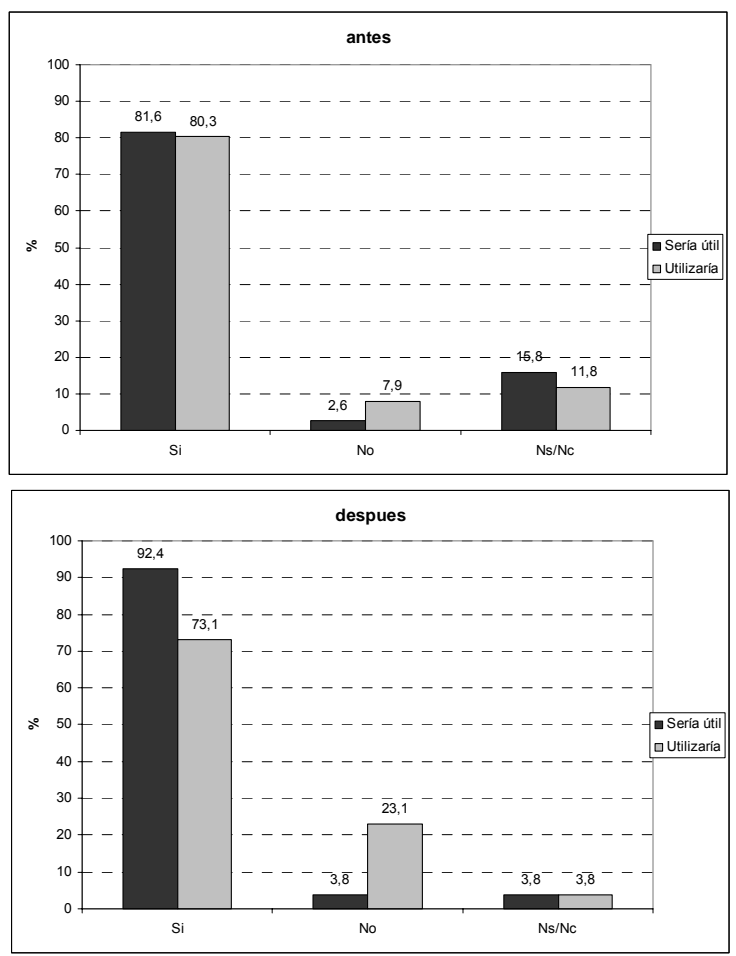

Figura 1. Actitud sobre el uso de instrumentos para evaluar la de Calidad de Vida Relacionada con la Salud:

Antes de Leer el SF-36 y después de leer el SF-36

Menos del $10 \%$ conoce algún instrumento de evaluación de la CVRS, la mayoría $(87,1 \%)$ nunca había completado un instrumento de evaluación de CVRS ni habían solicitado a sus pacientes que lo complete (93,5\%). Menos del $20 \%$ de los encuestados respondieron que no hablaban sobre CVRS o intentaban evaluarla en sus pacientes de alguna manera.

De los que evalúan la CVRS en sus pacientes la mayoría $(98,15 \%)$ lo hace subjetivamente, y no documenta esta información (89,3\%).

La Tabla 4 muestra las actitudes de los farmacéuticos encuestados sobre la evaluación de la calidad de vida relacionada con la salud y la práctica farmacéutica; y en la tabla 5 se muestran las opiniones de los farmacéuticos encuestados sobre los instrumentos de evaluación de la calidad de vida relacionada con la salud y la practica farmacéutica.

Las principales barreras y/u obstáculos para evaluar la CVRS según los encuestados son: la falta de instrumentos validados, falta de tiempo del paciente, el miedo del paciente de la invasión de su privacidad, que el paciente no pueda llenar cuestionarios y el tiempo requerido para que los pacientes completen el instrumento (tabla 6). El tiempo considerado necesario para que los pacientes completen un cuestionario de CVRS fue de 16,8 minutos $(8,59)$.
El $46 \%$ reconoce la semejanza de las preguntas del MOS SF 36 con las que formulan a sus pacientes en la evaluación de la CVRS.

El 76,9\% considera que la evaluación de la CVRS en el seguimiento farmacoterapéutico es importante.

Los Comentarios sobre la CVRS o la encuesta realizada se dividen en a favor $(80 \%)$ y en contra $(20 \%)$ de la CVRS y la práctica farmacéutica:

A. A favor: Sobre los instrumentos de evaluación de la CVRS: "Un instrumento sistemático para evaluar la CVRS sería útil en la practica...". Sobre evaluación de la CVRS: "Es una buena idea evaluar objetivamente la CVRS. La evaluación de la CVRS sería útil en la educación sanitaria y en el seguimiento farmacoterapéutico. La CVRS se evalúa a diario y no queda constancia...".

B. En contra: "Es un procedimiento engorroso y concepto difícil de ver objetivamente...hay que tener muchos conocimientos y estar perfectamente entrenado".

\section{DISCUSIÓN}

Los resultados sugieren que los farmacéuticos no están familiarizados con el concepto de CVRS que aparece en la literatura médica, el hallazgo de que el concepto de CVRS no estuvo presente en los estudios universitarios, según la mayoría de los encuestados, sugiere un déficit en la formación profesional mas aun ahora que se esta impulsando la realización del seguimiento farmacoterapéutico en las farmacias comunitarias y los resultados que se esperan obtener con el seguimiento farmacoterapéutico es mejorar la calidad de vida relacionada con la salud del paciente. Sería muy importante tener una metodología para evaluar estos resultados, ya que los farmacéuticos perciben la necesidad de tener un conocimiento adicional de la CVRS para evaluarla en sus pacientes. Los farmacéuticos indicaron que su conocimiento para evaluar la CVRS en sus pacientes debería ser de "mucho conocimiento" (38,5\%), esta realidad no es diferente el estudio realizado por Bentley y col en los Estados Unidos de Norteamérica donde los farmacéuticos calificaron su conocimiento actual de la CVRS mucho menor del nivel necesario para poder evaluarla. Además los comentarios de los encuestados también revelaron que ese entrenamiento y educación es un problema que necesita considerarse. Varios comentarios de los encuestados ilustran el ánimo por parte de farmacéuticos para recoger información de CVRS. Aunque después de recibir y leer el instrumento SF36 , los farmacéuticos siguen opinando que sería útil $(92,4 \%)$, el porcentaje disminuye un poco en si lo utilizarían $(73,1 \%)$, y esta disminución puede deberse a que después de leerlo consideren que necesitan mas información y entrenamiento adicional para poder aplicarlo. 
Tabla 4. Actitud sobre la Calidad de Vida Relacionada con la Salud y la práctica farmacéutica

\begin{tabular}{|c|c|c|c|c|c|}
\hline \multicolumn{2}{|c|}{ Opinión sobre CVRS y practica farmacéutica } & \multicolumn{3}{|c|}{ Escala (\%) } & Otros(\%) \\
\hline \multicolumn{2}{|c|}{$\begin{array}{l}\text { La CVRS es un objetivo importante de la atención } \\
\text { farmacéutica }\end{array}$} & $7(42,1)$ & $5(30,3)$ & $6(19,7)$ & 7,9 \\
\hline \multicolumn{2}{|c|}{ Los farmacéuticos son capaces de evaluar la CVRS } & $4(23,7)$ & $5(21,1)$ & $7(21,1)$ & 34,1 \\
\hline \multicolumn{2}{|c|}{$\begin{array}{l}\text { Se necesita entrenamiento adicional } \\
\text { objetivamente la CVRS }\end{array}$} & $7(42,1)$ & $6(30,3)$ & $5(18,4)$ & 9,2 \\
\hline $\begin{array}{l}\text { La CVRS es una cara } \\
\text { no puede ser medida, }\end{array}$ & $\begin{array}{l}\text { terística subjetiva y personal que } \\
\text { terpretada o comparada }\end{array}$ & $3(28,9)$ & $1(27,6)$ & $2(19,7)$ & 23,8 \\
\hline $\begin{array}{l}\text { La información de la } \\
\text { proveerle mejor atenci }\end{array}$ & $\begin{array}{l}\text { VRS de un paciente ayudaría para } \\
\text { n }\end{array}$ & $7(43,4)$ & $5(29)$ & $6(21)$ & 6,6 \\
\hline \multirow{3}{*}{$\begin{array}{l}\text { Las medidas clínicas } \\
\text { como la presión } \\
\text { arterial o colesterol } \\
\text { sérico son mas: }\end{array}$} & Útiles que la CVRS & $3(40,8)$ & $4(27,3)$ & $5(13,2)$ & 22,3 \\
\hline & Importantes que la CVRS & $3(38,2)$ & $4(38,2)$ & $2(9,2)$ & 14.4 \\
\hline & fáciles de obtener que la CVRS & $7(31,6)$ & $6(27,6)$ & $5(26,3)$ & 14,5 \\
\hline
\end{tabular}

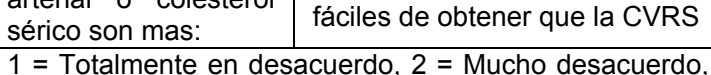

1 = Totalmente en desacuerdo, 2 = Mucho desacuerdo, $3=$ En desacuerdo, $4=$ Algo de acuerdo, $5=$ De acuerdo, $6=$ Muy de acuerdo, 7 = Sumamente de acuerdo

\begin{tabular}{|c|c|c|c|c|}
\hline \multirow{2}{*}{$\begin{array}{l}\text { Opiniones } \\
\text { Esta información mejoraría la habilidad de comunicarme con los } \\
\text { pacientes. }\end{array}$} & \multicolumn{3}{|c|}{ Escala (\%) } & \multirow{2}{*}{ Otro(\%) } \\
\hline & $3(30,8)$ & $4(26,9)$ & $5(42,3)$ & \\
\hline $\begin{array}{l}\text { Los pacientes aportarían información en esta forma, que } \\
\text { típicamente no me darían. }\end{array}$ & $2(23,1)$ & $3(38,5)$ & $4(23,1)$ & 15,3 \\
\hline $\begin{array}{l}\text { La información dada por este instrumento es difícil de entender e } \\
\text { interpretar. }\end{array}$ & $1(42,3)$ & $2(23,1)$ & $3(23,1)$ & 11,5 \\
\hline Los pacientes no podrían completar este instrumento & $1(34,6)$ & $2(23,1)$ & $3(26,9)$ & 15,4 \\
\hline Estarían más satisfechos con el servicio que mi farmacia provee & $3(30,8)$ & $4(34,6)$ & $5(23,1)$ & 11,5 \\
\hline Los pacientes serían más adherentes con su tratamiento & $2(11,5)$ & $3(30,8)$ & $4(50)$ & 7,7 \\
\hline $\begin{array}{l}\text { EL instrumento sería útil para le cribado con la que detectaría } \\
\text { cuando aconsejar a los pacientes que vayan a su médico }\end{array}$ & $3(42,3)$ & $4(38,5)$ & $5(19,2)$ & 0 \\
\hline $\begin{array}{l}\text { Este instrumento sería un buen método para medir cambios en } \\
\text { la CVRS de mis pacientes }\end{array}$ & $3(23,1)$ & $4(46,2)$ & $5(26,9)$ & 3,8 \\
\hline
\end{tabular}

\begin{tabular}{|l|l|l|l|l|}
\hline \multicolumn{4}{|l|}{ Tabla 6. Barreras y/u obstáculos que tienen los farmacéuticos para evaluar la CVRS } \\
\hline \multicolumn{3}{|l|}{ Oscala $(\%)$} & - & 38,4 \\
\hline Falta de tiempo del farmacéutico & $3(30,8)$ & $4(30,8)$ & $5(26,9)$ \\
\hline Falta de instrumentos de CVRS validados & $3(15,4)$ & $4(38,5)$ & 19,2 \\
\hline Falta de tiempo del paciente & $3(34,6)$ & $4(38,5)$ & - & 26,9 \\
\hline Desgano del paciente para llenar cuestionarios & $3(34,6)$ & $5(34,6)$ & - & 30,8 \\
\hline A los farmacéuticos no les gusta reunir datos de CVRS & $2(38,5)$ & $3(34,6)$ & - & 26,9 \\
\hline $\begin{array}{l}\text { Falta de habilidad del farmacéutico para interpretar y } \\
\text { utilizar datos de CVRS de pacientes }\end{array}$ & $3(26,9)$ & $4(30,8)$ & $1(26,9)$ & 15,4 \\
\hline El miedo del paciente de la invasión de su privacidad & $3(23,1)$ & $4(38,5)$ & $5(23,1)$ & 15,3 \\
\hline La incapacidad del paciente para llenar cuestionarios & $2(30,8)$ & $3(15,4)$ & $4(34,6)$ & 19,2 \\
\hline $\begin{array}{l}\text { No existe remuneración potencial para esta actividad } \\
\text { El tiempo requerido para que los pacientes completen el } \\
\text { instrumento }\end{array}$ & $1(30,8)$ & $4(26,9)$ & $2(19,3$ \\
\hline $\begin{array}{l}1 \text { = Ningún obstáculo, 2 = Obstáculo pequeño, 3 = Algo de obstáculo, } 4=\text { Mucho Obstáculo, } 5=\text { Obstáculo } \\
\text { excesivo }\end{array}$ & $4(46,3)$ & - \\
\hline
\end{tabular}

Es esperanzador encontrar tantos farmacéuticos dispuestos a incorporar la información de CVRS en su práctica. Aunque la educación y entrenamiento permanecen como problemas superiores, sin una respuesta positiva por parte de los farmacéuticos la incorporación de información de CVRS en la práctica de la farmacia comunitaria no sería posible. Igualmente, todavía por ser determinado esta el hecho de cómo la información de CVRS puede usarse por los farmacéuticos para influir en los resultados. Sólo con farmacéuticos capacitados estas preguntas podrían ser contestadas.
Varios autores han sugerido que los farmacéuticos han estado desde hace mucho tiempo involucrados en la calidad de vida de sus pacientes. Los resultados tienden parcialmente a confirmar esta noción. Salek indica como un nuevo reto para los farmacéuticos el involucrase en este rol de dos maneras diferentes: mediante la medición y aplicación clínica de la metodología para medir el impacto de la enfermedad y el tratamiento en la CVRS del paciente mediante el uso de los instrumentos; y aconsejar a los pacientes e informar a los médicos acerca de la información disponible sobre impacto en la CVRS para los diferentes 
productos farmacéuticos, proveer información adicional importante para las decisiones clínicas y en la selección de la monitorización de fármacos. ${ }^{18}$ Las preguntas en el cuestionario SF-36 se parecen las preguntas que a veces hacen a sus pacientes proporciona adicional evidencia parcial de que los farmacéuticos están involucrados en la CVRS de sus pacientes. Se utilizó este cuestionario porque fue el más usado, según la literatura, por los farmacéuticos para evaluar la CVRS. La mayoría utilizan criterios subjetivos como la conversación con los pacientes. Estos resultados son similares a los obtenidos por Bentley, donde aproximadamente los dos tercios de la población encuestada utilizaba métodos subjetivos para evaluar la CVRS de sus paciente, esta situación puede deberse a la falta de familiaridad con los instrumentos de evaluación de CVRS. Los comentarios de los encuestados demostró la percepción de que los farmacéuticos actualmente (aunque no formalmente ni objetivamente) evalúan la CVRS de sus pacientes, mas aún los resultados evidencian que la información, aunque sea subjetiva, sobre la evaluación de la CVRS no se documenta $(89,3 \%)$, por falta de conocimiento de los instrumentos de evaluación; no existiendo constancia alguna de esta valiosa actividad realizada por el farmacéutico.

Según la definición de Atención Farmacéutica dada por el Documento de Consenso en Atención Farmacéutica auspiciado por el Ministerio de Sanidad y Consumo Español, y de la definición de "Pharmaceutical Care", la CVRS es un objetivo importante en la atención farmacéutica, los resultados demuestran que la mayoría de farmacéuticos esta sumamente de acuerdo con esta opinión.

Existen varios usos potenciales y beneficios de la evaluación de la CVRS en la práctica de la farmacia comunitaria. Los resultados muestran que un porcentaje elevado de farmacéuticos está deseoso de evaluar la CVRS en el seguimiento farmacoterapéutico. En general consideran a estos instrumentos útiles para complementar el seguimiento farmacoterapéutico, aunque para ello se necesitaría un entrenamiento y capacitación adicional, para usarlos eficazmente.
Estos resultados muestran la existencia de un posible rol del farmacéutico comunitario en la evaluación de la CVRS especialmente en enfermedades crónicas que afecten significativamente la CVRS, lo que puede ser incluido en el seguimiento farmacoterapéutico y así valorar los resultados en la mejora de calidad de vida, como se enuncia en la definición de Atención Farmacéutica.

Los efectos positivos de los servicios farmacéuticos en la salud de los pacientes deberán seguir incorporando medidas de CVRS como resultado, complementado con indicadores clínicos, económicos y humanísticos.

\section{CONCLUSION}

Existen varios usos potenciales y beneficios de la evaluación de la CVRS en la práctica de la farmacia comunitaria. En su práctica profesional los farmacéuticos evalúan de manera subjetiva la CVRS de sus pacientes. Los resultados muestran que un porcentaje elevado de farmacéuticos está deseoso de evaluar la CVRS en el seguimiento farmacoterapéutico. En general consideran a estos instrumentos útiles para complementar el seguimiento farmacoterapéutico, aunque para ello se necesitaría un entrenamiento y capacitación adicional, para usarlos eficazmente.

Estos resultados muestran la existencia de un posible rol del farmacéutico comunitario en la evaluación de la CVRS especialmente en enfermedades crónicas que afecten significativamente la CVRS, lo que puede ser incluido en el seguimiento farmacoterapéutico y así valorar los resultados en la mejora de calidad de vida, como se enuncia en la definición de Atención Farmacéutica.

\section{AGRADECIMIENTOS}

Con el apoyo del Programa Alßan, Programa de becas de alto nivel de la Unión Europea para América Latina, $n^{\circ}$ de identificación E03D09244PE"

\section{Referencias}

1. Dauphinee-Wood S.Assessing Quality of Life in Clinical Research: From where have we come and where are we going? J Clin Epidemiol 1999; 52(4): 355-63.

2. Testa M, Simonson D. Assessment of quality-of-life outcomes. N Engl J Med 1996; 334 (13): 835-40.

3. Sullivan P, Wu A. Quality of life and HIV: Current assessment tools and future directions for clinical practice. AIDS Read 2003;13(12):583-97.

4. Soto Álvarez J Incorporación de estudios de calidad de vida relacionada con la salud en los ensayos clínicos: bases y recomendaciones prácticas. Uso de una lista-guía para su correcto diseño y/o evaluación An. Med. Interna (Madrid) 2003; 20(12): 633-44

5. Alonso J. La medida de la calidad de vida relacionada con la salud en la investigación y la práctica clínica. Gac Sanit 2000;14(2):163-7.

6. Martin Zurro A, Cano Perez JF. Atención Primaria. Conceptos, organización y práctica clínica. 5ta Edición. p250

7. Lohr KN, Aaronson NK, Alonso J, Burnam MA, Patrick DL, Perrin EB, Roberts JS.. Evaluating quality of life and health status instruments: development of scientific review criteria. Clin Ther 1996; 18: 979-92.

8. Ventegodt S, Merrick J, Andersen N. Meassurement of Quality of Life IV. Use of the SEQOL, QOL5, QOL1, and other global and generic questionaires. TheScientificWordIJOURNAL 2003;3:992-1001 
9. Velarde-Jurado E, Ávila-Figueroa C. Consideraciones metodológicas para evaluar la calidad de vida. Salud Publica Mex 2002;44:448-463.

10. Anguita, JC, Labrador JRR, Candel JP. Medidas de calidad de vida relacionada con la salud. Conceptos básicos, construcción y adaptación cultural. Med Clín (Barc) 2001; 116(20): 798-6.

11. Hepler CD, Strand LM. Opportunities and responsibilities in pharmaceutical care. Am J Hosp Pharm. 1990; 47(3): 53343.

12. Dirección General de Farmacia y Productos Sanitarios. Consenso Español de Atención Farmacéutica. Ars Pharm 2001; 42: 221-41.

13. Kheir N, Emmerton L, Shaw J. Can pharmacists influence the health-related quality of life patients with asthma? Medical Sciences 2001;3(2):69-75

14. Bentley JP, Smith MC, Banahan III BF, Frate DA Parks BR. Quality of life assessment by community pharmacists: an exploratory study. Quality Life Res 1998;7(2): 175-86.

15. Pickard S. Johnsdon J, Farris KB. The impact of Pharmacists interventions on Health-related Quality of Life. Ann Pharmacother 1999;33:1167-72.

16. Kheir N, Foppe van Mill J, Shaw J, Sheridan J. Health-related quality of life measurement in pharmaceutical care. Targeting outcome that matters. Pharm World Sci 2004;26:125-8.

17. Zillich A, Ryan M, Adams A, Yeager B, Farris K. Effectiveness of a Pharmacist-based smoking-cessation program and its impact on quality of life. Pharmacotherapy 2002;22(6):759-65.

18. Salek M. Health-related quality of life measurement. A new Challenge for pharmacy practitioners. J Pharm Pharmacol 1993;45(suppl1):387-92. 